\title{
AVALIAÇÃO EXPERIMENTAL E NUMÉRICA DE DIFERENTES MODELOS DE COEFICIENTES DE ARRASTE ATRAVÉS DE ESPECTRO DE ENERGIA
}

\author{
D. WANKE, A. S.CORDEIRO,B.L. da SILVA, R. K. DECKER e H. F. MEIER \\ Universidade Regional de Blumenau, Departamento de Engenharia Química \\ E-mail para contato: diegownk@gmail.com
}

\begin{abstract}
RESUMO - Em colunas de bolhas, a principal força de interação entre a fase líquida e as bolhas é a força de arraste a qual pode ser modelada por meio de equações específicas. Neste sentido, propõe-se avaliar através da simulação numérica diferentes correlações para o coeficiente de arraste para o sistema bifásico ar-água, e comparar os resultados numéricos com resultados experimentais para os espectros de energia obtidos na unidade experimental de coluna de bolhas. Para viabilizar o emprego destes modelos de arraste foi necessário ainda determinar o diâmetro médio das bolhas, obtidas com a técnica de imagem por contraste para vazão de alimentação de ar de $5 \mathrm{~L} / \mathrm{min}$. Para validação dos resultados por meio da análise de seus respectivos espectros de energia, obtidos para a frequência de $1 \mathrm{~Hz}$, foi utilizado um sensor de pressão acoplado na parede da coluna de bolhas. As simulações numéricas foram realizadas com o software FLUENT 14.0 da ANSYS para três correlações diferentes, sendo duas já implementadas no software (Schiller-Naumann e Universal Drag) e uma trazida da literatura, proposta por Krishna e van Baten. A análise do espectro de energia indica que o modelo proposto na literatura se aproxima do resultado experimental como também o modelo de Schiller - Naumann para uma frequência de aquisição de dados de pressão de $1 \mathrm{~Hz}$.
\end{abstract}

\section{INTRODUÇÃO}

Reatores de coluna de bolhas são constituídos por vasos cilíndricos com um distribuidor de gás. O gás é alimentado na base da coluna, entrando em contato com a fase líquida, no formato de bolhas. A fase líquida pode conter sólidos em suspensão, para este caso o reator de coluna de bolhas é chamado de reator de coluna de bolhas de leito de lama, em inglês "slurrybubblecolumnreactors". Este tipo de reator multifásico promove um intenso contato entre as fases, intensificando assim os fenômenos de transferência de massa e calor (KANTARCIet al., 2005; URSEANU, 2013).

Há três regimes de operação básicos para coluna de bolhas, o regime homogêneo, regime heterogêneo e o regime heterogêneo "Slug". No primeiro regimeocorre a presença de bolhas pequenas e bem distribuídas ao longo da coluna, já no regimeheterogêneo pode-se observar bolhas com tamanhos maiores com zonas de recirculação e uma distribuição não uniforme das bolhas ao longo da coluna e por fim no regime heterogêneo tipo "Slug" as bolhas possuem aproximadamente o mesmo diâmetro da coluna de bolhas.Este regime apenas é observado em colunas de escala de bancada de 
menor diâmetro.

Segundo Cui e Fan (2004), uma maior compreensão da fluidodinâmica, especialmente da turbulência nesses sistemas, é necessária para melhorar os modelos de fechamento nos estudos de CFD (Fluidodinâmica computacional).

As estruturas turbulentas da fase líquida e como elas interagem com a fase gasosa são consideradas uma das mais importantes questões em escoamentos gás - líquido, visto que as interações entre as fases e as estruturas turbulentas afetam as características do escoamento, distribuição da fração volumétrica, queda de pressão e os mecanismos de transferência de massa, energia e quantidade de movimento. A turbulência gerada pelas bolhas ao ascenderem na coluna é denominado de turbulência induzida pelas bolhas. Já a turbulência gerada por uma grade é denominada de turbulência verdadeira (SATHE; JOSHI; EVANS, 2013; HOSOKAWA, TOMIYAMA, 2013).

Uma diferença notável entre os dois tipos de turbulência, induzido pelas bolhas e gerada por uma grade, é a inclinação do espectro de energia na região inercial. Um valor encontradopara a inclinação no caso da turbulência induzida pelas bolhas é de - 8/3, enquanto para a turbulência de grade esta inclinação é de - 5/3, fundamentado pela teoria de transferência de energia inercial de Kolmogorov(SATHE; JOSHI; EVANS, 2013). A Figura 1 apresenta o espectro de energia típico para fase líquida onde foram destacadas a região que contem energia (ou região de Grande Escala) e a região inercial (ou região de dissipação de Energia), como também a inclinação de - 5/3 na região inercial.

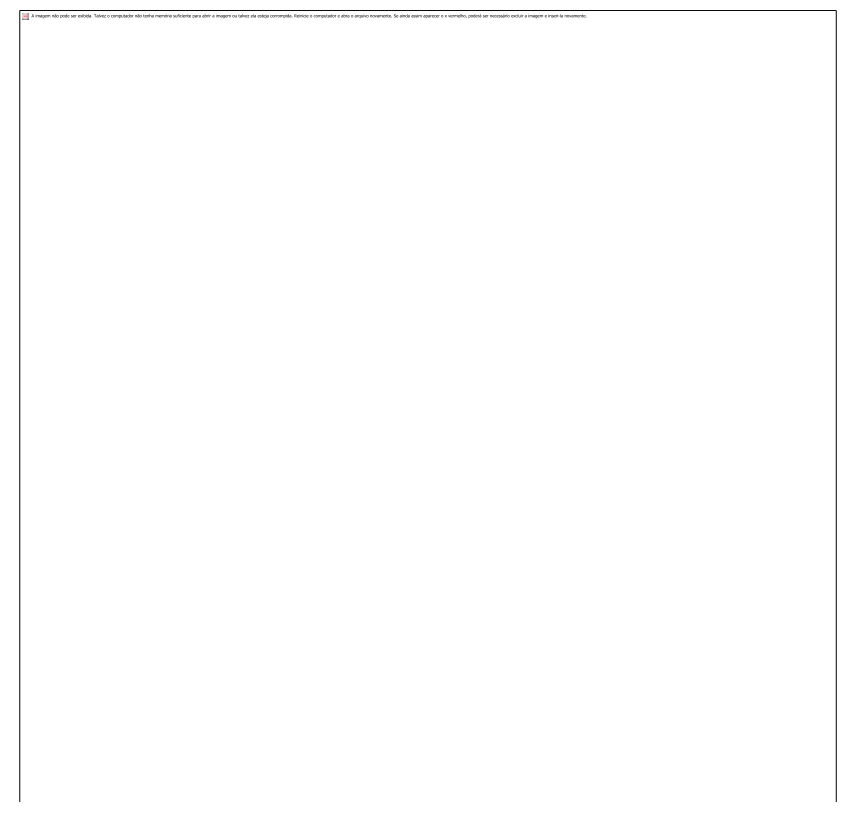

Figura 1 - Espectro de energia típico para fase líquida. 
Fonte: Adaptado de CUI; FAN, 2004, p. 1758.

Constatando assim, que a avaliação do espectro de energia pode ser uma ferramenta útil para validação de modelos numéricos, visto que esta é uma informação facilmente obtida experimentalmente por sondas de pressão, de alta frequência de aquisição de dados, e numericamente por sensores virtuais, podendo ser estes de pressão ou de velocidade.

Diante disto, tem-se como objetivo neste estudo avaliar através da simulação numérica diferentes correlações para o coeficiente de arraste para o sistema bifásico (ar - água), e comparar os resultados numéricos e experimentais para os respectivos espectros de energia.

\section{MATERIAIS E MÉTODOS}

A Unidade Experimental Coluna de Bolhas(UE-CB) é constituída por uma coluna de bolhas em acrílico, com $870 \mathrm{~mm}$ de altura e diâmetro interno de 144,2 mm. A coluna opera em sistema bifásico, gás - líquido, onde é utilizado água destilada para representar a fase líquida (fase contínua) e ar (fase dispersa) para representar a fase gasosa. A fase gasosa é injetada na base da coluna por um distribuidor de gás e ascende até o topo da coluna aberta ao ambiente. $\mathrm{O}$ distribuidor de gás é composto por um prato dispersor de ar (malha porosa), cujo diâmetro das perfurações é de $130 \mu \mathrm{m}$, com porosidade total de $20 \%$, resultando em uma área total de borbulhamento igual a $24,75 \mathrm{~cm}^{2}$. A Figura 2 apresenta um esquema da unidade experimental.

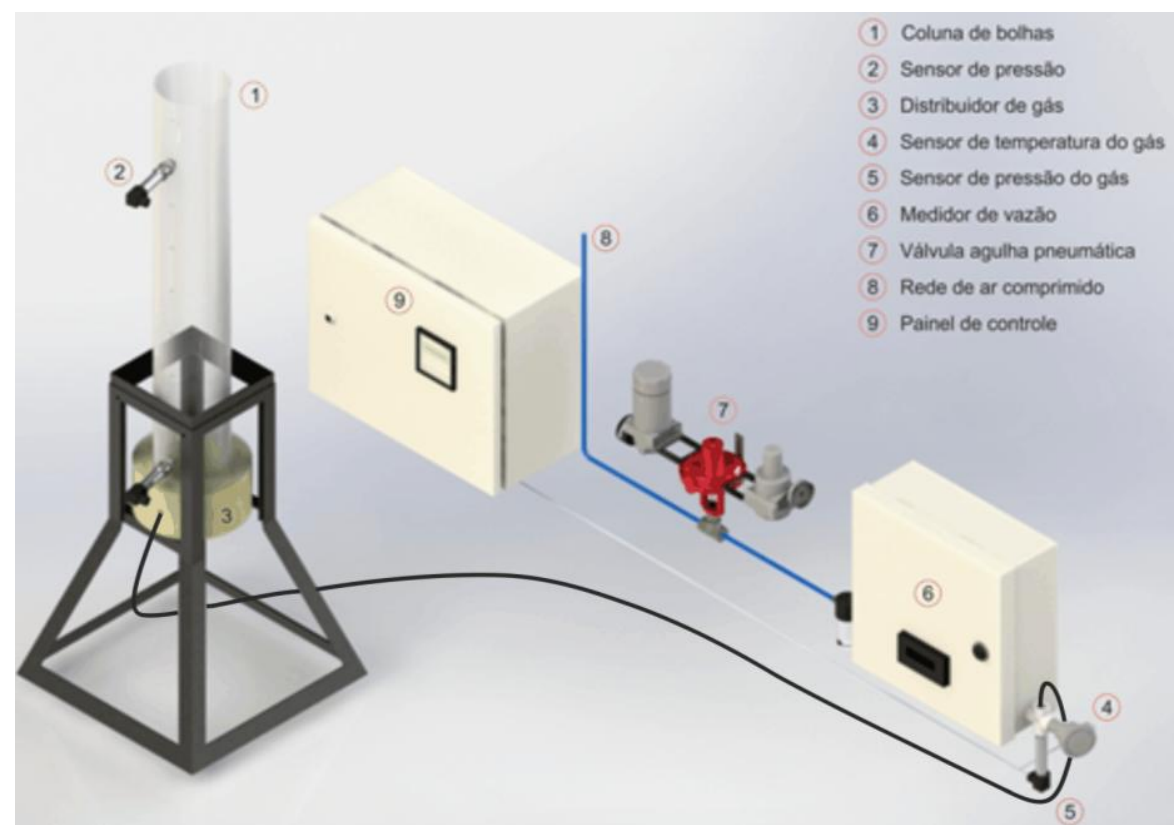

Figura 2 - Esquema Unidade Experimental - Coluna de Bolhas (UE-CB).

A transformada rápida de Fourier (FFT) é empregada na realização da distribuição da 
frequência de uma variável de interesse em relação a variação temporal, determinando assim o espectro de energia da variável correspondente. Os dados de pressão, descritos a seguir, são utilizados para o cálculo do espectro de energia empregando a técnica da FFT tanto para os dados de pressão numéricos como também para os dados de pressão experimentais.

\subsection{Experimentação Física}

Realiza-se a aquisição da pressão na coluna de bolhas, mediante utilização de transmissor de pressão,acoplado na UE-CB, na frequência de $1 \mathrm{~Hz}$ durante aproximadamente $10000 \mathrm{~s}$, para cálculo do espectro de energia experimental do escoamento borbulhante em coluna de bolhas. O primeiro ponto de monitoramento está localizado na parede e aproximadamente $418 \mathrm{~mm}$ da base da coluna. Já o segundo ponto está localizado também na parede e aproximadamente $18 \mathrm{~mm}$ da base da coluna, conforme Figura 3.

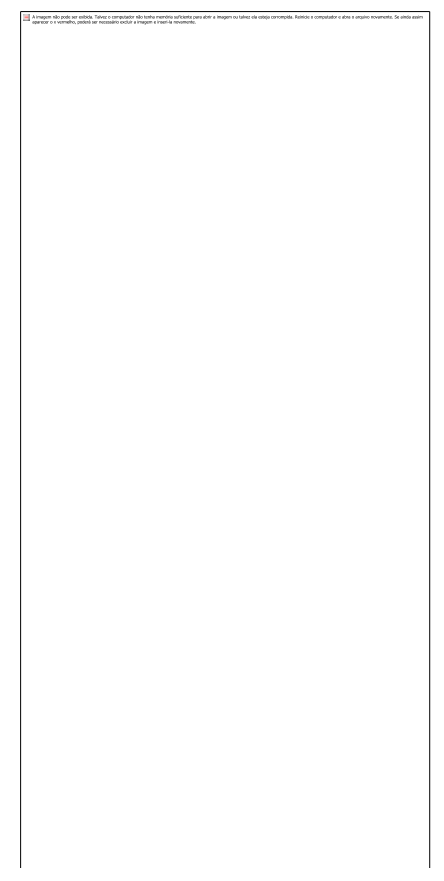

Figura 3 - Esquema sensores de pressão na coluna de bolhas.

\subsection{Simulação Numérica}

Para a realização da análise numérica inicialmente construiu-se a geometria e malha numérica da coluna de bolhas no programa ICEM 14.0 da ANSYS. A malha foi composta por elementos hexaédricos, totalizando 311.261 nós. Como pode ser observado na Figura 4, a região do dispersor de gases recebeu maior quantidade de pontos. 


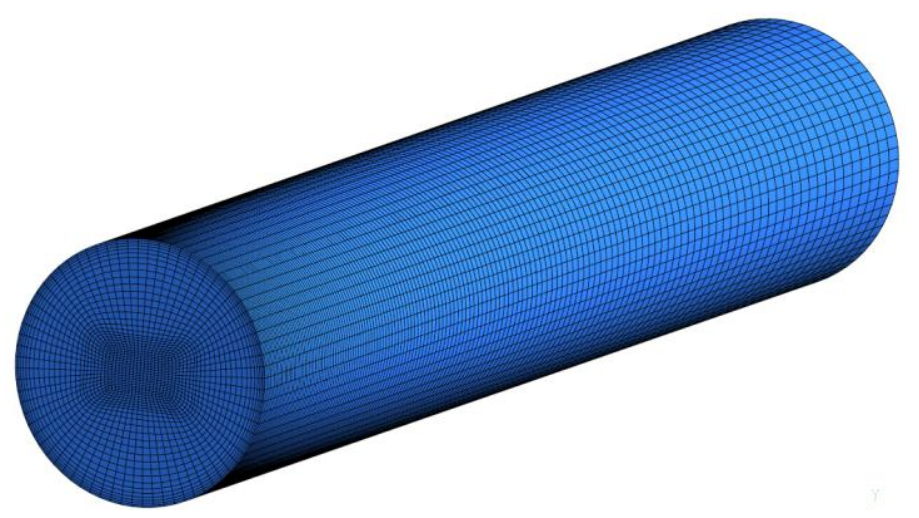

Figura 4 - Malha da coluna de bolhas.

Utiliza-se para o pré-processamento o programa FLUENT 14, da ANSYS, também utilizado para a execução das simulações, em estado transiente. Adota-se o modelo de turbulência k- $\varepsilon$ padrão, e as fases foram representadas através do modelo Euleriano - Euleriano, sendo a água a fase primária e o ar a fase secundária. Considera-se a força de arraste como predominante sobre as demais forças de interação interfaciais.

A análise numérica foi realizada com base na correlação para o coeficiente de arraste, com a aplicação dos modelos de Schiller-Naumann e Universal Drag, já implementados no programa, e a correlação proposta por Krishna, van Baten e Urseanu (2000). Os dois primeiros calculam o coeficiente de arraste como função do diâmetro médio das bolhas, obtido experimentalmente utilizando a técnica de imagem por contraste ou ainda em inglês "Shadowgraphy" na coluna de bolhas em estudo o qual foi de 2,28 mm. Já a correlação proposta por Krishna, van Baten e Urseanu (2000) independe do diâmetro da bolha.

A superfície que representa o dispersor de gases foi configurada como condição de contorno de entrada de ar, com a vazão mássica especificada como $0,00010208 \mathrm{~kg} / \mathrm{s}$, equivalente à condição de operação de 5 1/min da coluna real. A superfície do topo da coluna foi determinada como condição de contorno de pressão prescrita igual a zero.

A condição inicial foi especificada criando-se uma região com fração volumétrica de água igual a 1, para uma altura de colune de $50 \mathrm{~cm}$ a partir da base da mesma. A partir desta condição, executouse a simulação com passo de tempo de 0,001 segundo até que se completassem 20 segundos e o escoamento atingisse um estado aproximadamente estacionário. Deste ponto em diante, o passo de tempo foi reduzido para 0,01 segundo e os dados de pressão foram adquiridos a cada 1 segundo de simulação nos pontos de monitoramento, conforme os dados e condições estabelecidos nos experimentos físicos, ilustrados na Figura 5. 


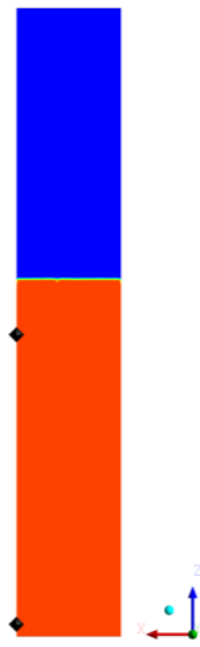

Figura 5 - Pontos de monitoramento de pressão.

\section{RESULTADOS E DISCUSSÕES}

Os resultados do cálculo do espectro de energia numérico e experimental, para cada um dos modelos propostos para o cálculo do coeficiente da força de arraste, são apresentados nas Figura 6, Figura 7 e Figura 8. Na Figura 6são apresentados os resultados dos espectros de energia para o modelo proposto por Krishna, van Baten e Urseanu (2000), na Figura 7o modelo de SchillerNaumann e por fim o modelo Universal Drga é apresentado na Figura 8.

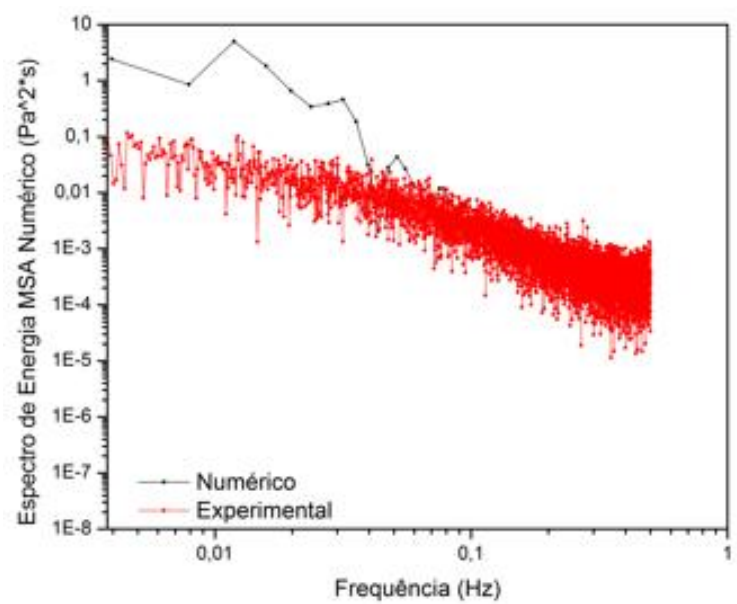

(A)

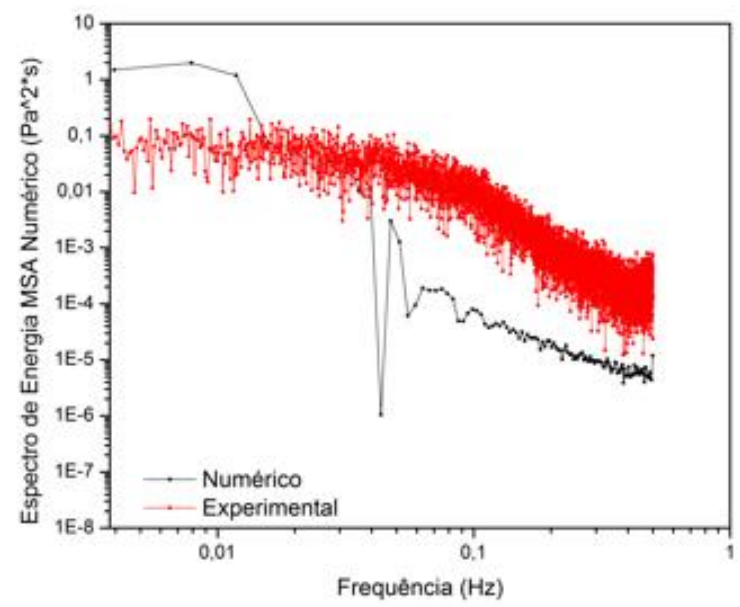

(B)

Figura 6 - Modelo proposto por Krishna van Baten e Urseano onde (A) base e (B) topo. 


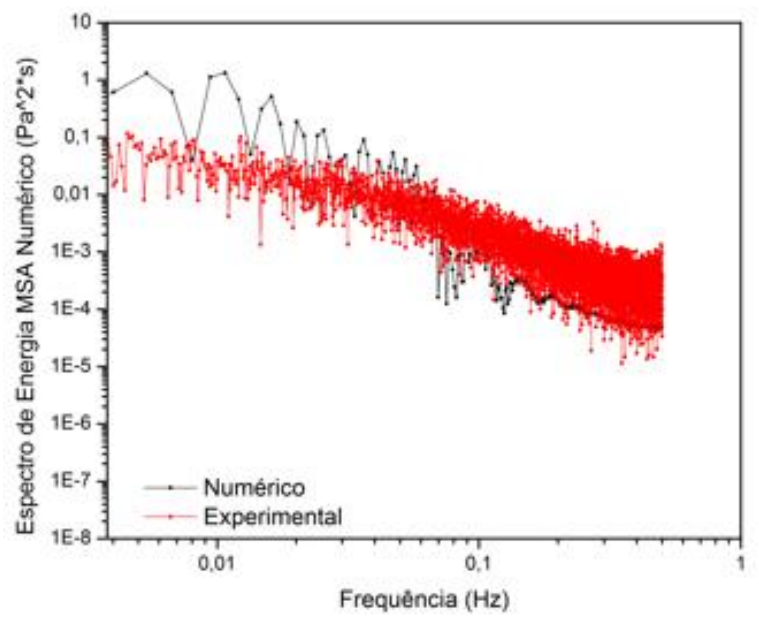

(A)

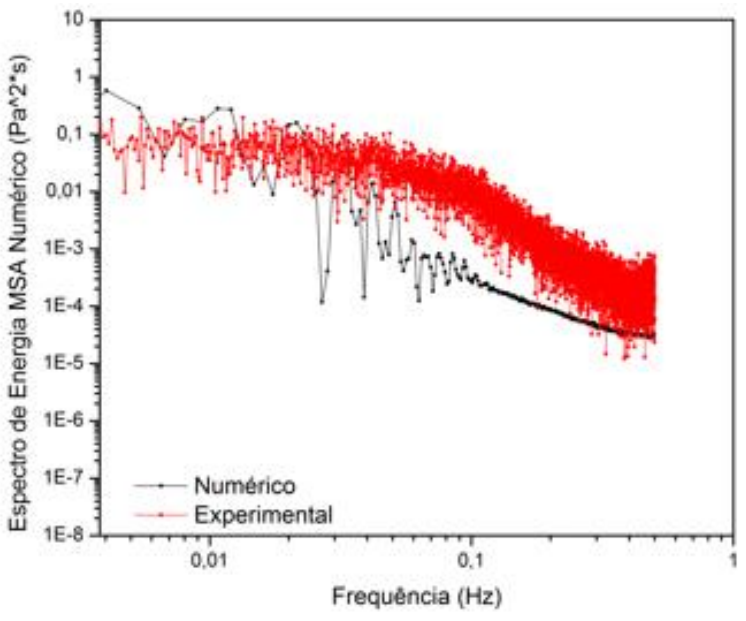

(B)

Figura 7 - Modelo de Schiller-Naumann onde (A) base e (B) topo.

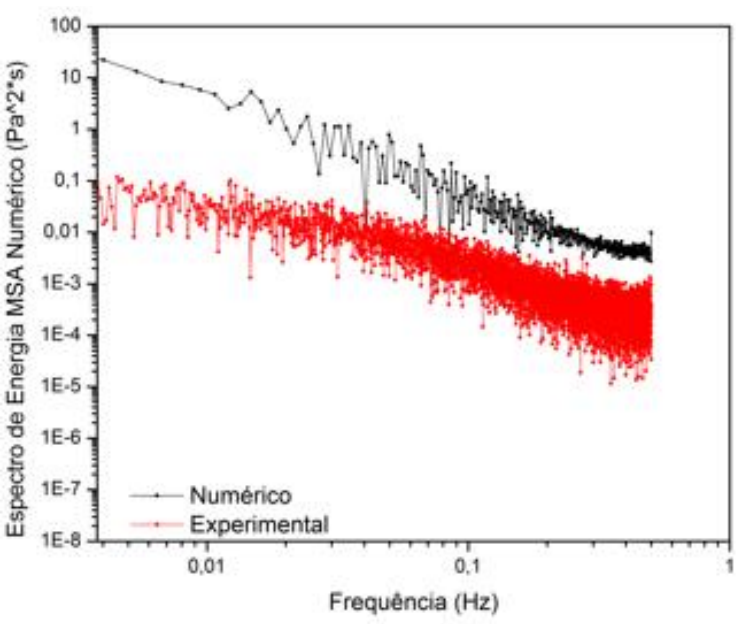

(A)

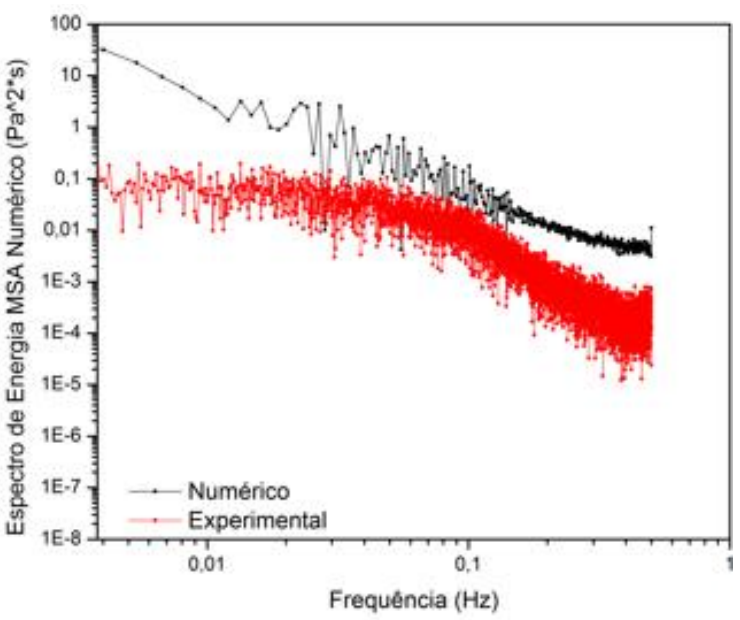

(B)

Figura 8 - Modelo Universal Drag onde (A) base e (B) topo.

Nota-se que tanto o modelo de Schiller - Naumann(Figura 7) quanto o modelo proposto por Krishna van Baten e Urseano (2000),Figura 6, apresentam resultados numéricos similares aos dados experimentais na base da coluna para frequência de $1 \mathrm{~Hz}$, mostrando assim uma validação qualitativa e quantitativa para região das grandes escalas. No entanto, quando os resultados obtidodos numericamente são comparados com os dados experimentais no topo da coluna, percebe-se que apenas o modelo para o coeficiente de arrasto de Schiller - Naumann foi capaz de prever quantitativamente a dinâmica do espectro de energia experimental por meio de modelo numérico. Já o resultado de Krishna van Baten e Urseano (2000) apresenta valores numéricos subestimados quando comparados aos resultados experimentais do espectro de energia no mesmo ponto. 
Por fim os resultados do espectro de energia obtidos com o modeloUniversal Drag (Figura 8) diferem quantitativamente dos dados experimentais tanto no topo quanto na base da coluna de bolhas, embora tenha apresentado informações qualitativas pertinentes.

\section{CONSIDERAÇÕES FINAIS}

O modelo proposto porSchiller - Naumann, para o cálculo do coeficiente de arraste, mostra ser adequado para a análise do escoamento em coluna de bolhas visto que os resultados obtidos numericamente mostram uma validade tanto quantitativa quanto qualitativa do espectro de energia, para uma frequência de $1 \mathrm{~Hz}$ na região das grandes escalas, nas duas posições avaliadas, quando comparados aos resultados experimentais do espectro de energia obtidos na mesma frequência. No entanto este modelo requer o conhecimento do diâmetro das bolhas, levando a utilização do modelo proposto por Krishna van Baten e Urseano (2000) quando este dado é dificilmente obtido.

Um estudo mais completo deve ser realizado com alta taxa de aquisição de dados, viabilizando assim o cálculo do espectro de energia completo para cada modelo empregado, possibilitando assim a avaliação da inclinação do espectro de energia na região inercial com os valores da literatura.

\section{REFERÊNCIAS}

CUI, Zhe; FAN, L. S..Turbulenceenergydistributions in bubblinggas - liquidandgas - liquid solidflow systems.Chem. Eng. Sc., n. 59, p.1755-1766, jan. 2004.

HOSOKAWA, Shigeo; TOMIYAMA, Akio. Bubble-inducedpseudoturbulence in laminar pipeflows.Int. J.ofHeatandFluidFlow, n. 40, p.97-105, fev. 2013.

KANTARCI, Nigar; BORAK, Fahir; ULGEN, Kutlu O. Bubblecolumnreactors.ProcessBiochem., n. 40, p.2263-2283, 2005.

KRISHNA, R.; VAN BATEN, J. M.; URSEANU, M. I. TrheephaseEuleriansimulationsofbubblecolumnreactorsoperating in thechurn-turbulent regime: a scaleupstrategy. Chem. Eng. Sc., n. 55, p. 3275-3286, 2000.

SATHE, Mayur; JOSHI, Jyeshtharaj; EVANS, Geoffrey. Characterizationofturbulence in rectangularbubblecolumn.Chem. Eng. Sc., v. 100, p.52-68, ago. 2013.

URSEANU, M.I.Scalingupbubblecolumnreactors.2000. f. Dissertação (Mestrado) - FacultyOf Science, Amsterdam, 2000. Disponível em: <http://dare.uva.nl/document/83975>. Acessoem: 27 fev. 2013. 